\title{
Monte Carlo Simulation of two-dimensional Kolmogorov flow
}

\author{
Jun Zhang and Jing Fan \\ Laboratory of High Temperature Gas Dynamics, Institute of Mechanics, \\ Chinese Academy of Sciences, Beijing 100190, China
}

\begin{abstract}
The two-dimensional Kolmogorov flow generated by an imposed unidirectional forcing varying sinusoidally in space is simulated by using direct simulation Monte Carlo method. Our simulation is performed under the condition of Knudsen number $\mathrm{Kn}=0.005$ and forcing wave number $k_{f}=4$. When the Reynolds number Re exceeds the critical value $\operatorname{Re}_{c}=\sqrt{2}$, the basic shear flow becomes instable and changes to cellular structures. As Re increases, two main regimes of the flow have been observed: small scale structure $\left(\operatorname{Re}_{c}<R e<7 \mathrm{Re}_{c}\right)$ and large scale structure $\left(\operatorname{Re}>7 \mathrm{Re}_{c}\right)$. When large scale structure is formed, the energy is inversely transferred from small scale forcing to large scale structure. As Re continues to increase, the evolution of flow field successively becomes quasi-periodical and irregular. This behavior provides a reasonable transition to turbulence with further increase of Re.
\end{abstract}

Keywords: Kolmogorov flow, Instability, Transition to turbulence, DSMC

PACS: 47.20.-k, 47.27.Cn, 47.11.Mn

\section{INTRODUCTION}

Kolmogorov flow, originally introduced by Kolmogorov in a seminar in 1959, has been widely used to study flow instability and transition to turbulence. Kolmogorov flow is generated by an imposed spatially periodic body forcing $\mathbf{F}=F_{0} \sin \left(k_{f} y\right) \hat{\mathbf{x}}$, where $F_{0}$ is the strength of the force field, $k_{f}$ is the wave number along the y direction, and $\hat{\mathbf{x}}$ is the unit vector in the $\mathrm{x}$ direction. For two-dimensional incompressible viscous flow, the corresponding Navier-Stokes equations is:

$$
\left\{\begin{array}{l}
\frac{\partial u}{\partial x}+\frac{\partial v}{\partial y}=0 \\
\frac{\partial u}{\partial t}+u \frac{\partial u}{\partial x}+v \frac{\partial u}{\partial y}=-\frac{1}{\rho} \frac{\partial p}{\partial x}+\eta \nabla^{2} u+F_{0} \sin \left(k_{f} y\right), \\
\frac{\partial v}{\partial t}+u \frac{\partial v}{\partial x}+v \frac{\partial v}{\partial y}=-\frac{1}{\rho} \frac{\partial p}{\partial y}+\eta \nabla^{2} v
\end{array}\right.
$$

Here $u$ and $v$ are the velocities in the $\mathrm{x}$ direction and $\mathrm{y}$ direction, respectively, and $\eta$ is the kinematic viscosity. The resulting basic flow $\mathbf{U}(y)=U \sin \left(k_{f} y\right) \hat{\mathbf{x}}$ is called Kolmogorov flow, where $U=F_{0} /\left(k_{f}{ }^{2} \eta\right)$, and the corresponding Reynolds number is $\mathrm{Re}=F_{0} /\left(k_{f}{ }^{3} \eta^{2}\right)$. The Kolmogorov flow has attracted a lot of theoretical and experimental studies. The theoretical studies of this problem have been mainly focused on the hydrodynamic stability viewpoint. Linear stability of the basic Kolmogorov flow was studied by Meshalkin and Sinai [1] and by Green [2]. They have shown that the flow becomes linearly unstable at a critical Reynolds number $\operatorname{Re}_{c}=\sqrt{2}$, beyond which a sequence of bifurcations will take place in the flow as Re number increases, and different flow patterns will be formed correspondingly. At sufficiently high Re number, the flow may become chaotic and turbulent. She [3], Platt et al. [4] have identified several scenarios of the transition to chaos and turbulence in

27th International Symposium on Rarefied Gas Dynamics, 2010

AIP Conf. Proc. 1333, 378-383 (2011); doi: 10.1063/1.3562678

(C) 2011 American Institute of Physics 978-0-7354-0888-0/\$30.00 
Kolmogorov flow by resorting to careful numerical simulations based on Navier-Stokes equations. On the experimental study side, Obukhov's review paper [5] discussed the laboratory realization of the Kolmogorov flow. More accurately, the instability of Kolmogorov flow has been examined in soap film by Burgess et al. [6].

In this paper we study the Kolmogorov flow via molecular simulation. Comparing with the continuum method, molecular simulation is a more direct and physical method. First, molecular method describes fluids on the most fundamental level. The dynamics of the molecules are explicitly calculated according to Newton's second law of motion, and fluid properties, such as the macroscopic velocity or temperature field, can be obtained as averages over the molecular information. Therefore, there is no need to assume the constitutive relation between the stress and strain in molecular simulation, while the constitutive relation should be assumed in continuum models. More important, fluctuations can arise spontaneously by thermal motions of molecules in molecular simulation, while they are usually lacking in continuum descriptions. It has been found that small fluctuations can be amplified by many orders of magnitude near the critical point, and then large fluctuations trigger the instability. Therefore, there is no need to add extra disturbances to induce instability in molecular simulation. The major drawbacks of molecular methods are the small length and time scales to which they are confined by their computationally-intensive nature. With the development of computational power, larger and larger molecular simulations can be performed.

In the recent two decades, molecular simulation has been applied to study the instability and transition to turbulence in various flows, such as Rayleigh-B é nard flow [7-10], Rayleigh-Taylor instability [11-13], etc. Meanwhile, Fluctuations have been shown to play an important role in the instability and transition [12, 14]. Therefore, it is very tempting to study Kolmogorov flow by using molecular simulation. In this paper, direct simulation Monte Carlo (DSMC) method [15] is used to simulate Kolmogorov flow. Our objective is to obtain a sequence of bifurcations as the Re number increases.

This paper is organized as follows. In the next section we describe the details of DSMC method in simulating Kolmogorov flow. Then, we present our simulation results and discuss the characteristics of different flow patterns. Concluding remarks is given at the end.

\section{DSMC METHOD FOR KOLMOGOROV FLOW}

DSMC method was introduced in the early 1960s to compute noncontinuum reentry flow fields that could not be obtained from approaches based on solving Navier-Stokes equations. The fundamental ideal of DSMC is to track a large number of representative molecules, with their motions and intermolecular collisions assumed uncoupled within small time intervals. Molecular motions are modeled deterministically, while molecular collisions are treated statistically. With the development of computational power, DSMC has been applied to complex flows in the continuum regime.

Here two-dimensional Kolmogorov flow is studied by using DSMC method. The simulation domain is square, that is, $L_{x}=L_{y}=800 \lambda$, where $\lambda$ is the mean free path of gas molecules. Periodic boundary conditions are assumed in both directions. The flow is maintained through an external force field $\mathbf{F}=F_{0} \sin \left(2 \pi k_{f} y / L_{y}\right) \hat{\mathbf{x}}$. Throughout our study the underlying forcing wave number is taken to be $k_{f}=4$. The Knudsen number can be defined as $\mathrm{Kn}=k_{f} \lambda / L_{y}=0.005$.

It should be noted that there is no energy exchange between the system and the boundaries in Kolmogorov flow because all of boundaries are assumed periodic. Therefore, if there is velocity gradient in the field due to external driving force, the temperature of the system will increase due to viscous dissipation. In order to obtain "stationary" nonequilibrium state, thermostat [16] should be used to prevent the temperature increasing. A simple method of controlling the temperature of a system in molecular simulation is velocity scaling. After each calculating step, the macroscopic velocity ( $\mathbf{U}$ ) and temperature $(T)$ of each sampling cell can be obtained by sampling the molecule information. We can control the temperature by adjusting the molecular velocity like the following equation:

$$
\left(\mathbf{c}^{\prime}-\mathbf{U}\right) /(\mathbf{c}-\mathbf{U})=\sqrt{\left(T_{0} / T\right)},
$$

where $\mathbf{c}$ and $\mathbf{c}^{\prime}$ are the original and new velocity of molecules, respectively, and $T_{0}$ is the desired temperature. In this way, the macroscopic velocity keeps invariant, but the temperature goes to the desired value.

Another way to control the temperature is to introduce a velocity dependent force on each molecule. The simplest choice seems to be a drag force proportional to the thermal velocity of molecules, that is, 
$\mathbf{F}^{\alpha}=-\alpha \mathbf{C}=-\alpha(\mathbf{c}-\overline{\mathbf{c}})$. The proportionality coefficient $\alpha$ is then adjusted to compensate for the heating. Based on Maxwell transport equation, we can obtain the energy equation as follows:

$$
\frac{3}{2} \rho R \frac{D T}{D t}=-P \frac{\partial \overline{c_{j}}}{\partial x_{j}}+\tau_{k j} \frac{\partial \overline{c_{k}}}{\partial x_{j}}-\frac{\partial q_{j}}{\partial x_{j}}+\rho \overline{F_{j} c_{j}}-\rho \overline{F_{j}} \cdot \overline{c_{j}}
$$

For incompressible viscous fluid, $\frac{\partial \overline{c_{j}}}{\partial x_{j}}=0$. If the temperature in the field keeps invariant and uniform, then $\frac{D T}{D t}=0$ and $\frac{\partial q_{j}}{\partial x_{j}}=0$. Consider the force terms in Eq. (3), for the external driving force $F^{e}$, because $F^{e}$ is not related to molecular velocity, $\overline{F_{j}{ }^{e} C_{j}}-\overline{F_{j}{ }^{e}} \cdot \overline{c_{j}}=0 ;$ for the velocity dependent drag force $F^{\alpha}, \overline{F_{i}^{\alpha}}=0$ and $\overline{F_{j}^{\alpha} C_{j}}=-\alpha \overline{C^{2}}=-\alpha \cdot 3 R T$ can be derived due to $F_{j}^{\alpha}=-\alpha C_{j}$. Therefore, Eq. (3) yields to:

$$
\tau_{k j} \frac{\partial \overline{c_{k}}}{\partial x_{j}}=\alpha \cdot 3 R T
$$

It means that the temperature increase is totally due to the viscous dissipation. Also, we can obtain the macroscopic velocity, temperature and stress term in each sampling cell after each calculating step. The velocity gradient can be identified by using least squares fitting method based on the molecular velocity and locations in each sampling cells. In this way, the proportionality coefficient $\alpha$ can be identified according to Eq. (4). We have tested the aforementioned two methods in simulating Kolmogorov flow. For the continuum regime Kn=0.005 interested here, both of the two methods can control the temperature and give comparable results.

\section{SIMULATION RESULTS}

In this section we present our simulation results of Kolmogorov flow. The length scale is normalized by mean free path of molecules. We have found that there are three different ranges of the flow patterns.

\section{Shear Flow below Re $_{c}$}

If the Re number is below the critical value $\sqrt{2}$, the basic Kolmogorov flow is stable. Figure 1 gives the distribution of horizontal velocity at the midst of $x$ direction along $y$ direction for $R e=0.83 \mathrm{Re}_{\mathrm{c}}$. It shows that the horizontal velocity varies sinusoidally in y direction, and the maximal speed is about $6.7 \mathrm{~m} / \mathrm{s}$, which is very close to the theoretical value $6.8 \mathrm{~m} / \mathrm{s}$ obtained by solving the Navier-Stokes equations.

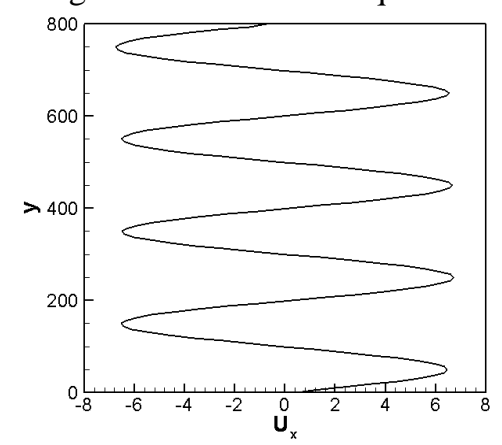

FIGURE 1. Distribution of horizontal velocity at the midst of $x$ direction along y direction for $\operatorname{Re}=0.83 \mathrm{Re}_{\mathrm{c}}$.

\section{Small Scale Structures between $\mathbf{R e}_{c}$ and $7 \mathbf{R e}_{\mathrm{c}}$}

In this range small cellular flow patterns are formed. Several characteristic flows are shown in Figs. 2-4. For $\operatorname{Re}=1.25 \mathrm{Re}_{\mathrm{c}}$ (Fig. 2), the flow is almost horizontal, and the saddle points of the separatrices of the streamlines are formed horizontally. For $\mathrm{Re}=2.08 \mathrm{Re}_{\mathrm{c}}$ (Fig. 3), the motion is mainly in the vertical direction, and the saddle points of the separatrix of the streamlines are joined vertically. This is in an agreement with the numerical results obtained by 
Platt et al. [4] based on Navier-Stokes equations under similar forcing. For $R e=3.33 R_{c}$, the flows still have the steady cellular structure but the number of vortices in the horizontal direction becomes double. This phenomenon is because that in this range the horizontal wave number of the fastest growing mode changes from 1 to 2 , which was first observed by Green [2] .

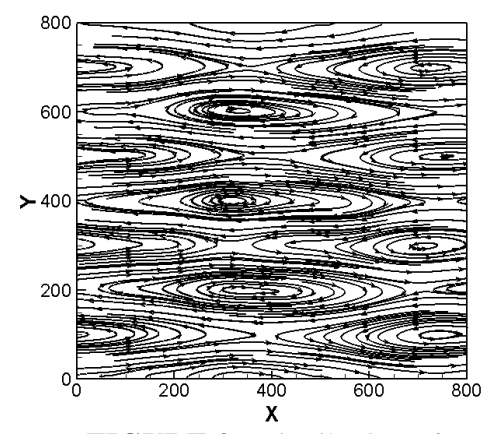

FIGURE 2. Distribution of streamlines for $\mathrm{Re}=1.25 \mathrm{Re}_{\mathrm{c}}$.

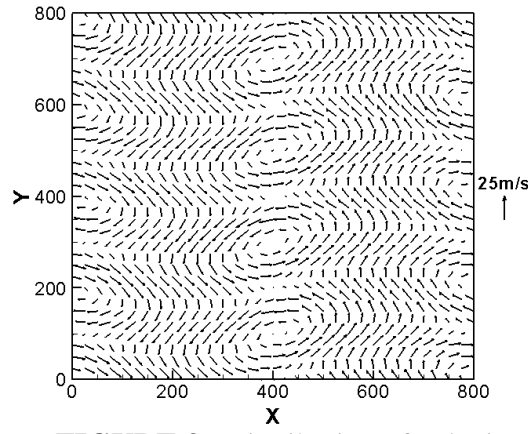

FIGURE 3. Distribution of velocity vectors for $\mathrm{Re}=2.08 \mathrm{Re}_{\mathrm{c}}$.

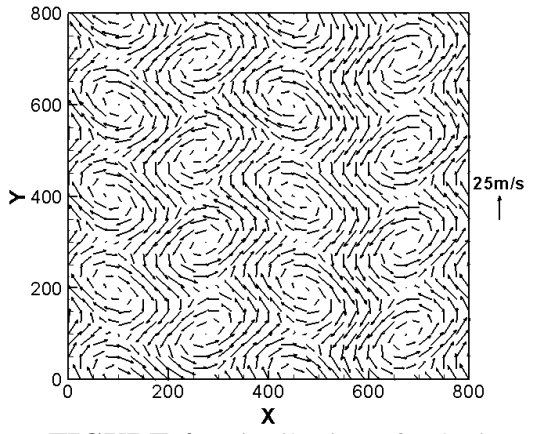

FIGURE 4. Distribution of velocity vectors for $\mathrm{Re}=3.33 \mathrm{Re}$.

As Re number increases, small amplitude perturbations at the saddle points of the streamlines will grow and lead to the breakdown of the symmetry of flow pattern. The velocity vector distributions for $\operatorname{Re}=4.17 \mathrm{Re}_{\mathrm{c}}$ is shown in Fig. 5. It clearly shows that the cellular vortices are no longer aligned on vertical lines and are of different size. However, the cellular structures are still steady.

As Re number continues to increase, the cellular structures become unsteady. We have observed this behavior by recording the evolution of power spectrum of velocity for $R e=6.25 R e_{c}$. Each sampling time $t_{s}$ is 1000 times of mean collision time of molecules. With the boundary conditions being assumed periodic, it is easy to determine the power spectrum of velocity $\left(\mathrm{P}\left(k_{x}, k_{y}\right)\right)$ by two-dimensional Fourier transformation on discrete space points. Here we focus on the power spectrum of horizontal velocity along $\mathrm{y}$ direction, and average is done along the $\mathrm{x}$ direction, that is, $\mathrm{P}\left(k_{x}=0, k_{y}\right)$. Figure 6 shows that both the power spectrum of $k_{y}=1$ and $k_{y}=4$ are changing with time, which represents that the flow is unsteady. Because the flow pattern for $R e=6.25 R_{c}$ is small scale cellular structure, the power spectrum of $k_{y}=4$ is larger than that of $k_{y}=1$.

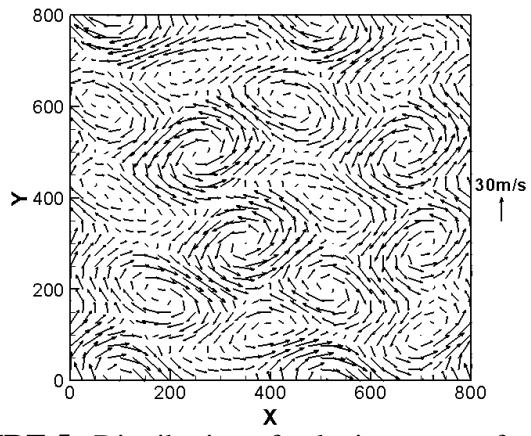

FIGURE 5. Distribution of velocity vectors for $R e=4.17 \mathrm{Re}_{\mathrm{c}}$.

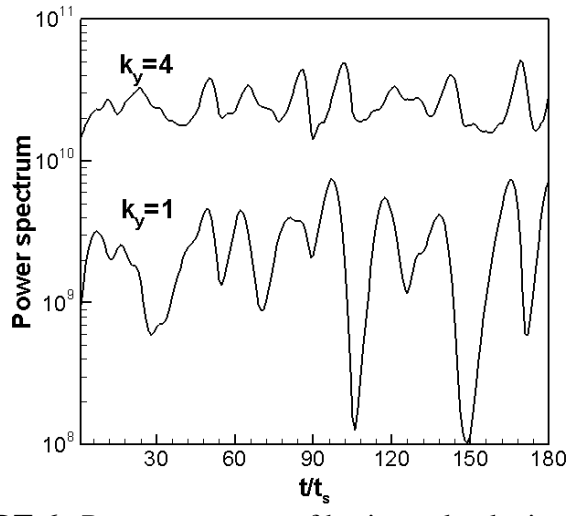

FIGURE 6. Power spectrum of horizontal velocity versus time for $\mathrm{Re}=6.25 \mathrm{Re}_{\mathrm{c}}$.

\section{Large Scale Structures above $\mathbf{7} \mathbf{R e}_{\mathrm{c}}$}

In this range, more large scale modes become linearly unstable; in addition, the interaction between different large scale modes becomes more efficient in fighting viscous dissipation. This can lead to new instabilities. For $\mathrm{Re}=8.33 \mathrm{Re}_{c}$, our simulation result shows that small vortices gradually mergers into large vortices in the long time transient period, and finally large scale structure (Fig. 7) is formed.

As Re number increases, the large scale structures become unsteady. A series of snapshots presented in Fig. 8 show the typical evolutions of the velocity vector field for $\mathrm{Re}=25.0 \mathrm{Re}_{\mathrm{c}}$. The sampling time $\mathrm{t}_{\mathrm{s}}$ is 1000 times of mean collision time of molecules. At $t=98 t_{s}$, there is large scale structure in the field, and then large scale structure 
gradually breaks up into small scale vortices. At $t=103 t_{s}$, the flow is mainly along the vertical direction, and two lines of four small vortices are aligned along vertical direction. This structure is also not steady, and small vortices merger into large vortices. At $t=110 t_{s}$, large structure is formed once again. It is interesting to note that small vortices in the inner of large structure are also not steady, and they oscillate like "breathing" continuously. Correspondingly, we give the evolution of power spectrum of horizontal velocity in Fig. 9. Also, average is done in the $\mathrm{x}$ direction. It clearly shows that the power spectrum of $k_{y}=1$ is larger than that of $k_{y}=4$. This is because that large scale structure has been formed in the field; hence the energy is inversely transport toward large scale from small scale forcing. This behavior is similar to the inverse cascade phenomenon has been reported in twodimensional turbulence $[17,18]$. Meanwhile, both power spectrum of $k_{y}=1$ and $k_{y}=4$ oscillate quasi-periodically. As the evolution from $t=98 t_{s}$ with large vortices to $t=103 t_{s}$ with small vortices, the power spectrum changes from a maximal value to a minimal value.

The evolution characteristic of flow patterns for $\mathrm{Re}=41.7 \mathrm{Re}_{c}$ is similar to that for $\mathrm{Re}=25.0 \mathrm{Re}_{c}$, but with more rapid changes between large scale structure and small vortices aligned along vertical direction. More important, it is obvious that the variance of power spectrum becomes more random and irregular for $\operatorname{Re}=41.7 \mathrm{Re}_{\mathrm{c}}$ (Fig. 10) than that for $\mathrm{Re}=25.0 \mathrm{Re}_{\mathrm{c}}$. This behavior provides a reasonable transition to chaos or turbulence with further increase of Re number.

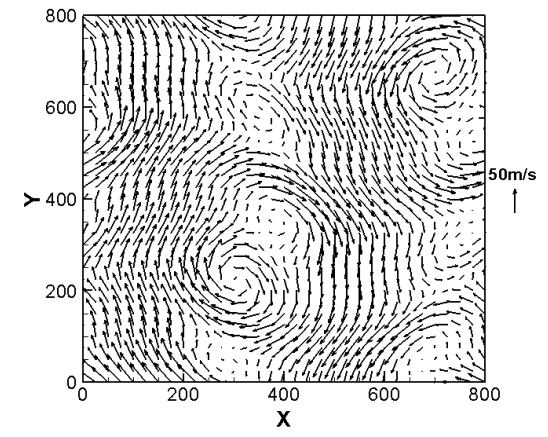

FIGURE 7. Distribution of velocity vectors for $\mathrm{Re}=8.33 \mathrm{Re}_{\mathrm{c}}$.

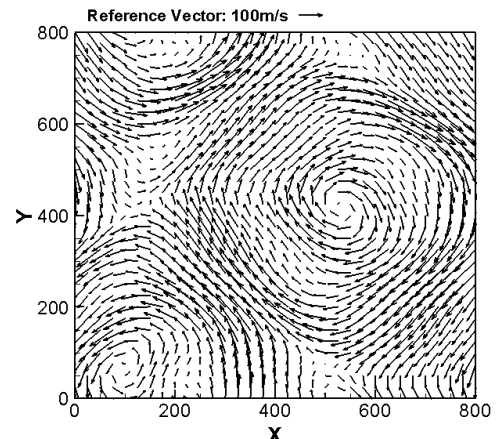

(a) $t=98 t_{s}$

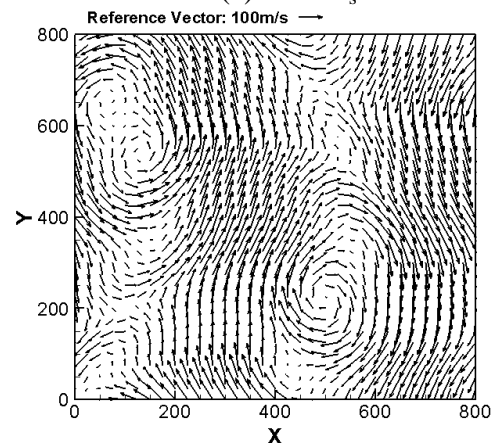

(d) $\mathrm{t}=107 \mathrm{t}_{\mathrm{s}}$

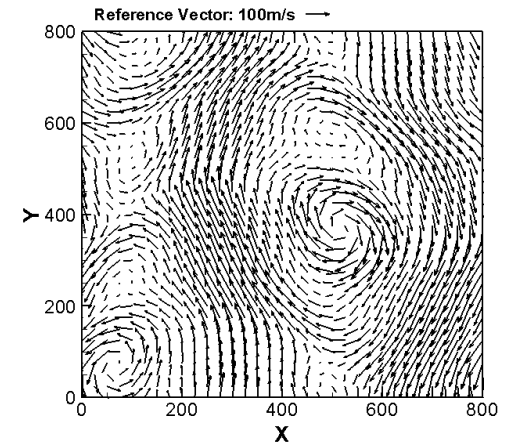

(b) $\mathrm{t}=101 \mathrm{t}_{\mathrm{s}}$

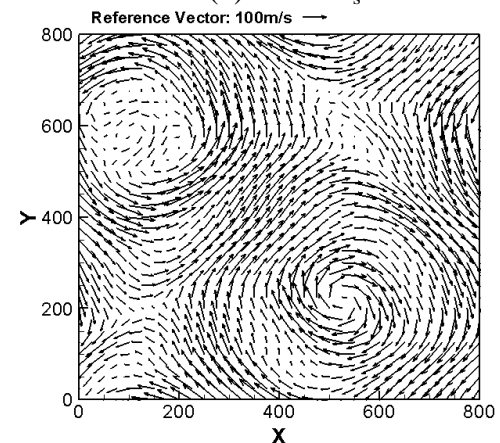

(e) $\mathrm{t}=110 \mathrm{t}_{\mathrm{s}}$

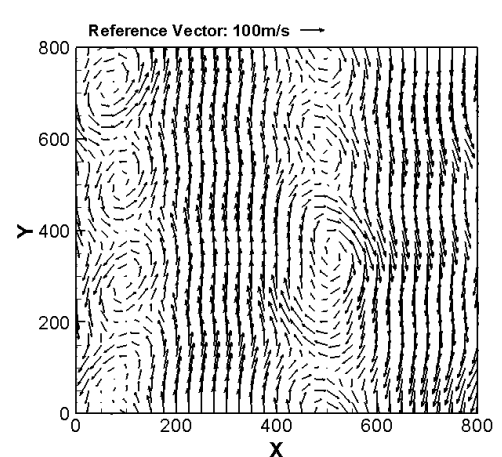

(c) $t=103 t_{s}$

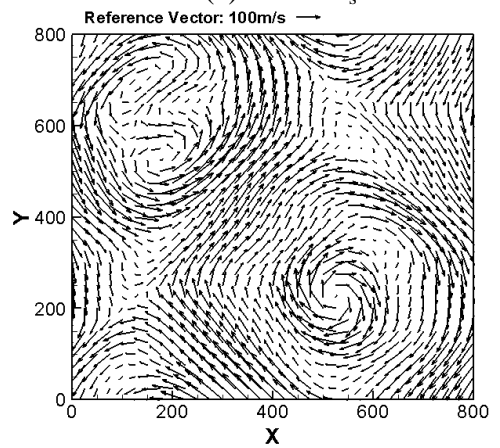

(f) $\quad t=115 t_{s}$

FIGURE 8. A series of snapshots of velocity vector field for $R e=25.0 R_{c}$. 


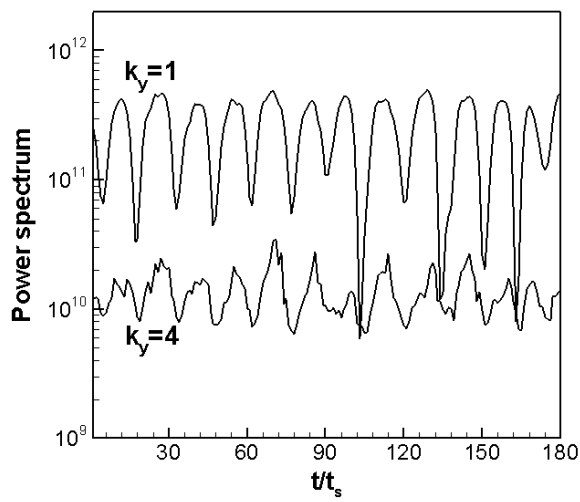

FIGURE 9. Power spectrum of horizontal velocity versus time for $\mathrm{Re}=25.0 \mathrm{Re}_{\mathrm{c}}$.

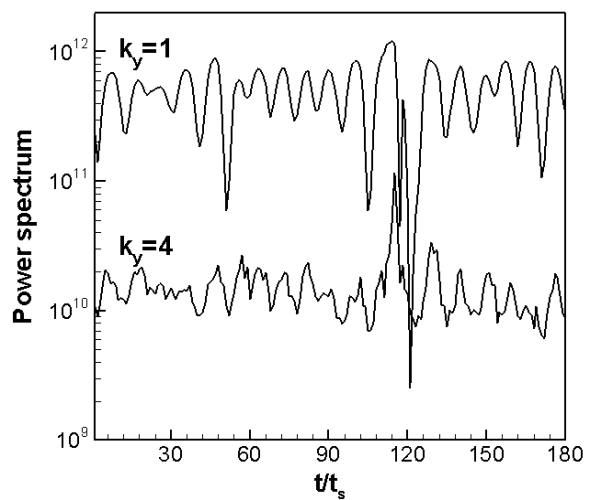

FIGURE 10. Power spectrum of horizontal velocity versus time for $\mathrm{Re}=41.7 \mathrm{Re}_{\mathrm{c}}$.

\section{CONCLUDING REMARKS}

In this paper, two-dimensional Kolmogorov flow is simulated by using DSMC method. Two main regimes of the flow have been observed: small and large scale structure regimes corresponding to different ranges of Re. Meanwhile, a sequence of bifurcations is shown to take place in each of the regimes of the flow. Our simulation is performed under the condition of $\mathrm{Kn}=0.005$, and thermostat is used to control temperature, so our simulations results are consistent with that obtained by solving incompressible viscous Navier-Stokes equations. Our further study will focus on the Kolmogorov flow over the whole range of the Knudsen number.

\section{ACKNOWLEDGMENTS}

This work was supported by the National Natural Science Foundation of China (Grant Nos. 10921062 and 11002147).

\section{REFERENCES}

1. L. D. Meshalkin and Y. G. Sinai, J. Appl. Math 25, 1700-1705 (1961).

2. J. S. A. Green, J. Fluid Mech. 62, 273-287 (1974).

3. Z. S. She, Physics Letters A 124, 161-164 (1987).

4. N. Platt, L. Sirovich, and N. Fitzmaurice, Physics of Fluids A 3, 681-696 (1991).

5. A. M. Obukhov, Russ. Math. Surv. 38, 113-126 (1983).

6. J. M. Burgess et al., Physical Review E 60, 715-721 (1999).

7. A. Garcia, "Hydrodynamic fluctuations and the direct simulation Monte Carlo method" in Microscopic Simulations of Complex Flows, edited by M. Mareschal, New York: Plenum, 1990, pp. 177-188.

8. E. Golshtein and T. Elperin, Journal of Thermophysics and Heat Transfer 10, 250-256 (1996).

9. S. Stefanov, V. Roussinov, and C. Cercignani, Physics of Fluids 14, 2255-2269 (2002).

10. J. Zhang and J. Fan, Chin. Sci. Bull. 54, 364-368 (2009).

11. K. Kadau et al., Proc. Natl Acad. Sci. 101, 5851-5855 (2004).

12. K. Kadau et al., Proc. Natl Acad. Sci. 104, $7741-7745$ (2007).

13. K. Kadau et al., Philosophical Transactions of the Royal Society A 368, 1547-1560 (2010).

14. J. Zhang and J. Fan, Physical Review E 79, 056302 (2009).

15. G. A. Bird, Molecular Gas Dynamics and Direct Simulation of Gas Flows, Oxford: Clarendon, 1994.

16. D. J. Evans and G. P. Morriss, Statistical mechanics of nonequilibrium liquids, London: Academic Press, 1990.

17. R. H. Kraichnan and D. Montgomery, Reports on Progress in Physics 43, 547-619 (1980).

18. P. Tabeling, Physics Reports-Review Section of Physics Letters 362, 1-62 (2002). 